\title{
Validación de un método cuantitativo de Evaluación de Riesgos de Trastornos Músculo-esqueléticos en las tareas de las artes plásticas a partir de un método cualitativo
}

\section{Validation of a quantitative method to assess the Risk of Musculoskeletal Disorders in the tasks of the plastic arts from a qualitative method}

\author{
Patricia Eugenia Sortillón-González ${ }^{1}$, Leonel Ulises Ortega-Encinas ${ }^{2}$, Jose Sergio-Lopez \\ Bojorquez $^{3}$, Julieta Amada Leyva-Pacheco ${ }^{4}$, Gerardo Ochoa-Salcido ${ }^{5}$ \\ ${ }^{1}$ Universidad Estatal de Sonora, Programa de Ingeniería Industrial en Manufactura, Ave Ley Federal del Trabajo, Colonia \\ Apolo, CP 83000 Hermosillo, Sonora, México \\ Corresponding author's e-mail: patricia.sortillon@ues.mx. https://orcid.org/0000-0002-9716-6693 \\ ${ }^{2}$ Universidad Estatal de Sonora, Programa de Ingeniería Industrial en Manufactura, Ave Ley Federal del Trabajo, Colonia \\ Apolo, CP 83000 Hermosillo, Sonora, México, e-mail: leonel.ortega@ ues.mx https://orcid.org/0000-0002-8054-917X \\ ${ }^{3}$ Universidad Estatal de Sonora, Programa de Ingeniería Industrial en Manufactura, Ave Ley Federal del Trabajo, Colonia \\ Apolo, CP 83000 Hermosillo, Sonora, México \\ e-mail: sergio.lopez@ues.mx https://orcid.org/0000-0002-6920-2829 \\ ${ }^{4}$ Universidad Estatal de Sonora, Programa de Ingeniería Industrial en Manufactura, Ave Ley Federal del Trabajo, Colonia \\ Apolo, CP 83000 Hermosillo, Sonora, México \\ e-mail: julieta.leyva@ues.mx https://orcid.org/0000-0001-7960-3848 \\ ${ }^{5}$ Instituto Tecnológico de Hermosillo, Programa de Ingeniería Industrial, Av. Tecnológico y periférico poniente s/n, \\ Colonia El Sahuaro, CP 83170 Hermosillo, Sonora. https://orcid.org/0000-0002-6232-7733
}

Autor de correspondencia

Patricia Eugenia Sortillón-González. patricia.sortillon@ues.mx

DOI https://doi.org/10.46589/rdiasf.vi35.338

Recibido 3 de febrero 2021

Aceptado 15 de mayo 2021

Publicado 30 de junio 2021.

\section{Resumen}

En años recientes, los trastornos músculo esqueléticos han recibido mucha atención, debido a su impacto en el desempeño y eficiencia laboral. Aunque los trastornos músculo esqueléticos están relacionados con los trabajadores industriales, algunos estudios revelan que son muchos otros sectores los que se ven afectados por ellos, sobre todo aquellos en donde se realizan tareas repetitivas, posturas sedentes y levantamiento de cargas. En las 
tareas de las artes plásticas prevalecen las tres condiciones mencionadas, de ahí el riesgo de presencia de trastornos músculo esqueléticos en los ejecutantes de las artes plásticas. En esta investigación se condujo un estudio longitudinal a lo largo de 16 semanas consecutivas en estudiantes de artes plásticas con el propósito de investigar el riesgo cualitativo de trastornos músculo esquelético durante el quehacer plástico y proponer un método cuantitativo para evaluar el riesgo de trastornos músculo esqueléticos en dichas tareas, se realiza la validación del método cuantitativo a partir del método cualitativo. Se aplicó un procedimiento estadístico de prueba de hipótesis para probar la significancia estadística de la recta de regresión lineal que correlaciona las valoraciones del método cuantitativo propuesto y el valor de índice RULA. Los resultados obtenidos demuestran que existe una relación lineal entre los dos valores, el cuantitativo y el cualitativo, por lo que el método cuantitativo propuesto es adecuado para evaluar el riesgo de trastorno músculoesquelético en las tareas de las artes plásticas. Los resultados obtenidos establecen un procedimiento cuantitativo en el que se consideran los factores antropométricos individuales de cada individuo, los cuales no están considerados en los procedimientos cualitativos.

Palabras clave: Arte, validación, Músculo-esquelético, Trastorno.

\begin{abstract}
In recent years, musculoskeletal disorders (MD) had received a lot of attention due to their impact on performance and work efficiency. Although musculoskeletal disorders are associated with industrial workers, some studies show that many other sectors are affected by them, especially those where repetitive tasks, sedent postures and load lifting are performed. In the arts plastic sector, the three conditions mentioned prevail, hence the risk of presence of musculoskeletal disorders in the art performers. In this research we conducted a longitudinal study over 16 consecutive weeks in plastic art students with the purpose of investigating the qualitative risk of musculoskeletal disorders during art tasks. A quantitative assessment was proposed to assess the risk of MD in plastic art tasks. The Rapid Upper Limb Assessment (RULA) which is a qualitative assessment method was used to validate the proposed cuantitative assessment. A statistical procedure of hypothesis testing was applied to prove the statistical significance of the linear regression line that correlates the proposed cuantitative risk value of MD and the RULA index value. The results obtained demonstrate that there is a linear relationship between the two variables, thus the cuantitative method is suitable for evaluating the risk of musculoskeletal disorder in the plastic arts tasks. The results obtained establish a quantitative procedure in which the individual anthropometric factors of each individual are considered of which qualitative methods are lacking.
\end{abstract}

Key words: art, validation, musculoskeletal, disorder. 


\section{Introducción}

La evidencia epidemiológica de los trastornos músculo esqueléticos es muy amplia y ha recibido mucha atención en años recientes, debido a su impacto en el desempeño y eficiencia laboral. Los trastornos músculo esqueléticos (TME) están asociados de acuerdo a Punnett (2004) a ciertos factores de riesgo que se pueden detectar en la actividad laboral y entre estos factores se encuentran a los movimientos repetitivos, a las posturas corporales no neutras y las cargas sostenidas. Los TME de acuerdo a Bernard (1997) incluyen un amplio rango de condiciones inflamatorias y denegerativas que afectan músculos, tendones, ligamentos, uniones, nervios periféricos y venas de soporte, incluyen síndromes clínicos tales como las inflamaciones de los tendones y condiciones relacionadas (epicondilitis y bursitis), desórdenes de compresión de nervios (síndrome del túnel de carpo, ciática) así como condiciones de mialgia, dolor de espalda baja y otros síndromes de dolor localizados no atribuibles a las patologías conocidas. Las regiones del cuerpo involucradas con los TME son la espalda baja, el cuello, el hombro, el brazo y la mano. Los TME de acuerdo a Rempel (1997) prevalecen en las ocupaciones de trabajo manual intenso tales como trabajo de oficina, servicio postal, limpieza, inspección y empaque industrial. En el ejercicio de las artes plásticas, en particular en la pintura, escultura y grabado se ejecutan movimientos y posturas que implican un trabajo manual intenso, con levantamientos de cargas, posturas de cuello y espalda fijas por periodos prolongados de dos hasta cuatro horas por tres días consecutivos en una semana, todas las cuales son factores de riesgo para el desarrollo de TME. Los TME son catalogados como enfermedades profesionales (Caban-Martinez et al.,2010) que aparecen en torno a los factores de riesgo asociados a ellos. Sin embargo, existe un desfase en la aparición de un trastorno y su método de detección, con frecuencia estos son detectados una vez que la enfermedad se hace manifiesta y presenta una sintomatología (Mitropoulos y Namboodiri, 2011). De acuerdo con Khlil en (Konz, 2008) en las sociedades industrializadas, el $80 \%$ de los adultos desarrollan problemas en la espalda. Según Bruce( 1997). De acuerdo a los datos presentados por Juno (2004) en el año de 2002 el Instituto Mexicano del Seguro Social (IMSS), reportó 387,806 accidentes y enfermedades de trabajo, de los cuales un bajo porcentaje corresponde a TME, sin embargo, la causa de este bajo porcentaje es que quienes los padecen, los reportan con hasta que los síntomas se hacen evidentes, las causas de esta baja prevalencia obedecen a deficiencias en el sistema de registro de las enfermedades. De acuerdo a Froines et al. (1986) la supervisión de las enfermedades ocupacionales no es específica y está desfasada en relación a la detección de la enfermedad, por lo cual no existen datos confiables y estadística de las mismas para lugares de trabajo específicos, esto se debe en gran parte a la falta de guías o pautas reconocer la enfermedad por una parte y por otra, debido que estas enfermedades responden a condiciones específicas. Las condiciones físicas de las tareas que se definen como factores de riesgo de acuerdo a Kolsch et al. (2003), son los patrones de movimiento 
repetitivo y descenso rápido de carga laboral, tiempo insuficiente de recuperación, levantamiento de cargas pesadas, esfuerzos manuales extremos, posturas no neutras tanto dinámicas como estáticas, concentraciones de presión mecánica, vibración completa o local del cuerpo, exposición local o completa del cuerpo al frío o cualquier combinación de estos factores presentes en el ambiente laboral en donde el trabajo exige una alta demanda.

En cuanto a los métodos de evaluación postural Sonne et al. (2012) presentó el desarrollo de una hoja de verificación de riesgos ergónomicos para oficinas, denominado ROSA (Rapid Strain Assessment) que fue diseñado para hacer una cuantificación rápida de los riesgos de asociados con el trabajo de computación para establecer un nivel de acción basado en el reporte del trabajador mismo. Los factores de riesgo se diagraman y codifican cuantitativamente y se emplea una correlación lineal para establecer la significancia entre las estaciones de trabajo consideradas. Esta evaluación fue considerada en un principio para ajustarse al proceso de diagnóstico de los factores de riesgo que pueden presentarse durante la actividad de pintura y grabado, pero se ha seleccionado al método RULA por ser las condiciones para las cuales fue concebido, similares a las que se llevan a cabo en el quehacer de las artes plásticas.

El confort postural (Kolsch et al., 2003) es definido como un conjunto de posturas que pueden ser adoptadas y movimientos que pueden ser llevados a cabo sin conducir a otros movimientos secundarios, con el fin de compensar a los primeros. Un individuo en estado de confort postural no deseará compensar una postura o un movimiento. El confort no puede ser medido directamente o por medio de cuestionarios, existen varios métodos para evaluar la sensación de no confort, entre ellos el método de Corlett (Corlett et al, 1976), en el cual los participantes indican las partes del cuerpo donde sienten no confort y establecen un valor de severidad del mismo, sin embargo, estos métodos tiene una naturaleza subjetiva en sus resultados y por otra parte el cuestionario conduce a incrementar la sensación de no confort experimentada con lo cual los resultados pueden tener un sesgo.

Zhang et al (1986) señalan que una biomecánica pobre puede convertir el confort en no confort, aun cuando exista una buena biomecánica ésta no es una condición necesaria y suficiente para el confort. El confort ya ha sido definido en varias investigaciones; en este sentido Slater(1985) en (De Looze et al 2003) señalan al confort como un estado placentero de armonía psicológica, fisiológica y física entre el ser humano y su ambiente. En estas investigaciones se realizan diferentes asociaciones hipotéticas con diversos factores, para después determinar las relaciones entre las mediciones objetivas y las subjetivas del confort y el no confort. Se emplea como método de medición del no confort la distribución de presión, la que a su vez se puede asociar con un grado de replicación importante hacia las mediciones subjetivas. Para las demás variables, como son el perfil de la espina dorsal o la actividad muscular, las asociaciones reportadas son poco claras y no son estadísticamente significativas.

El método de medición de presión por medio de sensores resulta ser un método de validación para el índice de riesgo de TME, sin embargo, no se ha escogido para 
validar el método cuantitativo que se propone en esta investigación, debido a que no está disponible localmente el equipo para realizar esas mediciones.

Con el propósito de seleccionar las mediciones de confort que son significativamente asociadas a la percepción se emplea un modelo de regresión. El modelo de regresión arroja como predictor significativo de confort al pico de presión y se valida al índice al no poderse rechazar la hipótesis de correlación entre el índice y la percepción del confort. Sin embargo, debido a que los datos fueron influenciados por las condiciones experimentales y la variabilidad antropométrica, en el mismo documento se menciona el beneficio de realizar nuevas investigaciones, como método para refinar el valor del índice.

En 2011 se emitió un artículo titulado "Validation of a new index for seat confort assessment base objetive and subjetive measurements" (Lanzotti, 2011) en el que se valida un índice estadístico denominado Pérdida de confort por prorrateo de presión, el cual es empleado para evaluar el confort de un asiento; esta validación se realiza en función de la relación entre las mediciones subjetivas de confort y las mediciones objetivas de confort, a través de una comparación, este índice incluye el pico de presión que es empleado como uno de los índices para evaluar el confort de los asientos. las mediciones subjetivas se obtienen a partir de cuestionarios, y las mediciones objetivas se realizan a través de un tapete con sensores de presión con diferentes variables.

En esta investigación se utilizan los resultados de la evaluación postural del método RULA para cada sujeto a fin de validar el método cuantitativo para evaluar el riesgo de TME que se propone.

El uso de los resultados subjetivos cómo método para comparar a una variable cuantificada como lo es la del método que se propone es una estrategia que está validada por un procedimiento estadístico, el valor de esta estrategia incide también en el hecho de que se le está utilizando un valor de referencia que está vinculado a lo que un individuo puede sentir y expresar verbalmente.

En 1996, Robert Marley y Nirmal Kumar, desarrollan un proceso de evaluación del confort músculo-esquelético, el cual es una adaptación de análisis ergonómico de tipo cuestionario, que es contestada por los propios evaluados y que son en total 797 empleados de una compañía. Los resultados se analizan para determinar si la evaluación realizada por los mismos afectados, adecuadamente predice si un empleado tendrá algún tratamiento médico debido a un no confort relacionado con la actividad laboral. Se emplea un análisis discriminante para mostrar que las cuantificaciones obtenidas pueden identificar a quien ha recibido un tratamiento. Se determina una frecuencia y un nivel de no confort, a través de los cuales se establecen zonas de alta probabilidad, baja probabilidad y no probabilidad de tener un tratamiento. Aunque el método no es una herramienta de diagnóstico, puede emplearse como un instrumento de identificación de problemas ergonómicos en el sistema músculo-esquelético. Varios autores (Hamberg-van Reenen et al, 2008) establecen la premisa de que existe una relación entre la sensación de no confort y los trastornos músculo-esqueléticos y que éstos últimos afectan de alguna manera la sensación de confort que podemos experimentar. En diferentes estudios de medicina (Tilley, 2001) se establece que las uniones del cuerpo tienen condiciones 
específicas de reposo, en donde los músculos están completamente relajados o con un nivel mínimo de esfuerzo y que estas condiciones particulares de las uniones, generan configuraciones geométricas que corresponden a posiciones de reposo de las uniones. Galinsky et al (2001), indican que esta posición de reposo minimiza el trastorno músculo-esquelético y optimiza la percepción del confort.

En general, las uniones del cuerpo, definen debido al movimiento que pueden tener, un cierto rango de movimiento, usualmente medido de forma angular, en el cual se puede considerar que existe una condición de reposo y a su vez de confort, si ese rango de movimiento se sobrepasa entonces, se pueden experimentar una percepción de no confort, y a su vez la posibilidad, de un daño músculo-esquelético, cuando existe una repetición del movimiento. De esta forma una unión puede quedar representada por una variable, a la que se le puede denominar rango de movimiento, y que define los límites en los cuales esa unión se moverá sin causar una percepción de no confort. Apostólico et al (2013) presentan un procedimiento estadístico para determinar los rangos de movimiento del cuello, hombro, codo, muñeca y tobillo, que consiste en obtener los ángulos de rotación de la articulación bajo los principios ergonómicos conocidos y en los cuales el sujeto experimenta una sensación de confort. Se emplea este procedimiento para obtener la función de probabilidad que describe el comportamiento estadístico de los datos y así establecer los límites entre los cuales un sujeto experimenta confort al rotar una articulación

En esta investigación se determinan los valores límites superior e inferior de movimiento de los segmentos corporales, para los cuales el sujeto experimenta confort al realizar el movimiento y mantener la postura, que según Galinsky(2000) minimiza la aparición del trastorno músculo esquelético. Se emplea el modelo biomecánico estático para el plano sagital y se calculan las cargas y momentos de fuerza respecto de la unión o centro de rotación del tronco-muslo, considerando el peso de los segmentos corporales y el que añaden las cargas externas soportadas. El análisis se realiza en aquellas tareas que tienen mayor repetición y condiciones de carga. Se plantea un modelo biomecánico estático de una postura de reposo para los dos extremos de confort (rotación mínima y máxima del segmento corporal en la que se experimenta confort) y otro para la condición real. Se propone una valoración cuantitativa de TME que es un índice agregado no ponderado, para los momentos de fuerza respecto al centro de rotación tronco-muslo en la postura de confort mínima y máxima y en base a los momentos de fuerza para la postura real. El análisis de momentos de fuerza se realizó en base al modelo biomecánico postural estático para el plano sagital de las tareas realizadas por los estudiantes de la licenciatura en Arte de la Universidad de Sonora en las áreas de pintura, escultura y grabado.

\section{Metodología}

\subsection{Diseño del estudio y participantes}


Se realizó un estudio prospectivo y observacional durante 16 semanas consecutivas en los semestres impares, correspondientes al primero, tercero, quinto y séptimo semestres de la licenciatura en artes plásticas de la Universidad de Sonora, en las áreas de pintura, escultura y grabado. En esta investigación, la población estudiada comprendió 186 estudiantes distribuidos en 11 grupos: grupo de pintura I (28 alumnos), grupo de escultura I(30 alumnos), grupo de grabado I (27 alumnos), grupo de pintura III (23 alumnos), grupo de escultura III (25 alumnos), grupo de grabado III (23 alumnos), grupo de pintura específico I (10alumnos), grupo de escultura específico I (4 alumnos), grupo de grabado específico I (5 alumnos), grupo de pintura específico III (6 alumnos) y grupo de escultura específico III (5 alumnos). Los estudiantes participantes tuvieron un horario escolar de 7:00 a.m. a 3:00 p.m. en una base de cinco días continuos por semana. Fueron excluidos de este estudio aquellos participantes en estado de preñez o bajo tratamiento médico. Los datos requeridos para el estudio fueron colectados durante el periodo matutino. Los participantes de este estudio fueron informados de los objetivos del mismo y los que decidieron participar firmaron un consentimiento de participación. Los participantes tuvieron la oportunidad para decidir renunciar a su participación en el estudio en cualquier momento, y en los casos así previstos, les fueron entregados sus resultados.

\subsection{Procedimiento}

\subsubsection{Evaluación RULA}

Una vez firmados los consentimientos de participación por parte de los sujetos de estudio, se recabaron los datos sociodemográficos de todos los mismos, como son la edad, sexo, estado civil y laboral. Se realizó un proceso de observación de las posturas adquiridas por los estudiantes al realizar las tareas de pintura, escultura y grabado, con el fin de seleccionar aquellas actividades en donde existían factores de riesgo para TME, como son la repetición de movimientos, posturas sedentes y levantamiento de cargas. Para las tareas que cumplieron con factores de riesgo asociados, fue aplicada la evaluación RULA (Rapid Upper Limb Assessment) y la valoración cuantitativa propuesta, a fin de determinar el indice RULA y establecer el riesgo de TME en las tareas desarrolladas por los pintores, los escultores y los grabadores y la correspondiente valoración cuantitativa. El programa de aplicación de la evaluación RULA, se muestra en la Tabla No. 1

Tabla No. 1 Programa de Evaluación de Riesgos de TME por el Método RULA y Valoración Cuantitativa

\begin{tabular}{|c|c|c|c|c|c|c|c|c|c|c|c|c|c|c|c|c|c|c|}
\hline & & & \multicolumn{16}{|c|}{ Semanas } \\
\hline Grupos & Semestre & Cantidad & 1 & 2 & 3 & 4 & 5 & 6 & 7 & 8 & 9 & 10 & 11 & 12 & 13 & 14 & 15 & 16 \\
\hline Pintura I & 1 & 28 & 2 & 2 & 2 & 2 & 2 & 2 & 2 & 1 & 2 & 2 & 2 & 2 & 1 & 2 & 1 & 1 \\
\hline Escultura I & 1 & 30 & 2 & 2 & 2 & 2 & 2 & 2 & 2 & 2 & 1 & 1 & 2 & 2 & 2 & 2 & 2 & 2 \\
\hline
\end{tabular}


Grabado I

Pintura III

Escultura III

Grabado III

Pintura Específico I

Escultura Específico I

Grabado Específico I

Pintura III Específico

Escultura III Especifico

27
23
25
23
10
4
5
6
5

$\begin{array}{ll}1 & 1 \\ 1 & 2 \\ 2 & 1 \\ 2 & 1 \\ & 1 \\ 1 & 1 \\ & \\ 1 & 1 \\ 1 & 1\end{array}$

$\begin{array}{llllll}2 & 2 & 2 & 2 & 2 & 2 \\ 1 & 1 & 1 & 1 & 1 & 1 \\ 1 & 1 & 1 & 2 & 2 & 1 \\ 1 & 1 & 2 & 1 & 2 & 1 \\ 1 & 1 & 1 & 1 & & \\ 1 & & & & & \\ & & 1 & 1 & 1 & 1 \\ 1 & 1 & 1 & & & \end{array}$

El programa de evaluaciones con el método RULA y la valoración cuantitativa propuesta comprende 16 semanas consecutivas y once grupos de estudiantes, correspondientes al primer semestre de estudios, los grupos pintura I, escultura I y grabado I; al tercer semestre los grupos de pintura III, escultura III y grabado III; para el quinto semestre los grupos específico pintura I, específico escultura I y específico grabado I y para el séptimo semestre específico pintura III y Específico escultura III. Las evaluaciones fueron aplicadas semanalmente en las asignaciones indicadas en la tabla.

\subsubsection{Modelo Biomecánico Coplanar estático}

Se estableció un modelo biomecánico coplanar para la parte sagital derecha del cuerpo, de acuerdo a lo indicado en las figuras No. 1 y No. 2 bajo la premisa de que el cuerpo humano está conformado por una serie de uniones rígidas denominadas segmentos, caracterizados por su masa, peso y centro de gravedad, dichos segmentos están unidos por centros de rotación que corresponden en anatomía a las articulaciones corporales. Durante la ejecución de una tarea, los segmentos corporales experimentan una rotación angular, siendo el ángulo de rotación del segmento corporal otra de las variables del modelo biomecánico. El modelo propuesto es un sistema bidimensional estático en el que se representan las fuerzas y momentos de fuerza que actúan sobre las diferentes articulaciones del cuerpo durante la ejecución de una tarea. 


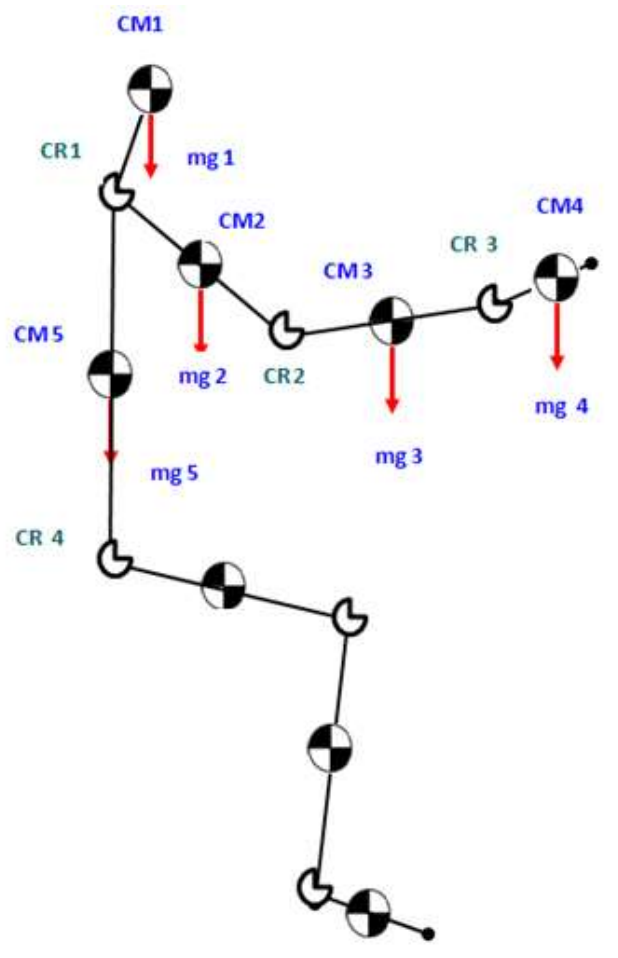

Figura No. 1 Modelo Biomecánico Coplanar para Pintura y Grabado

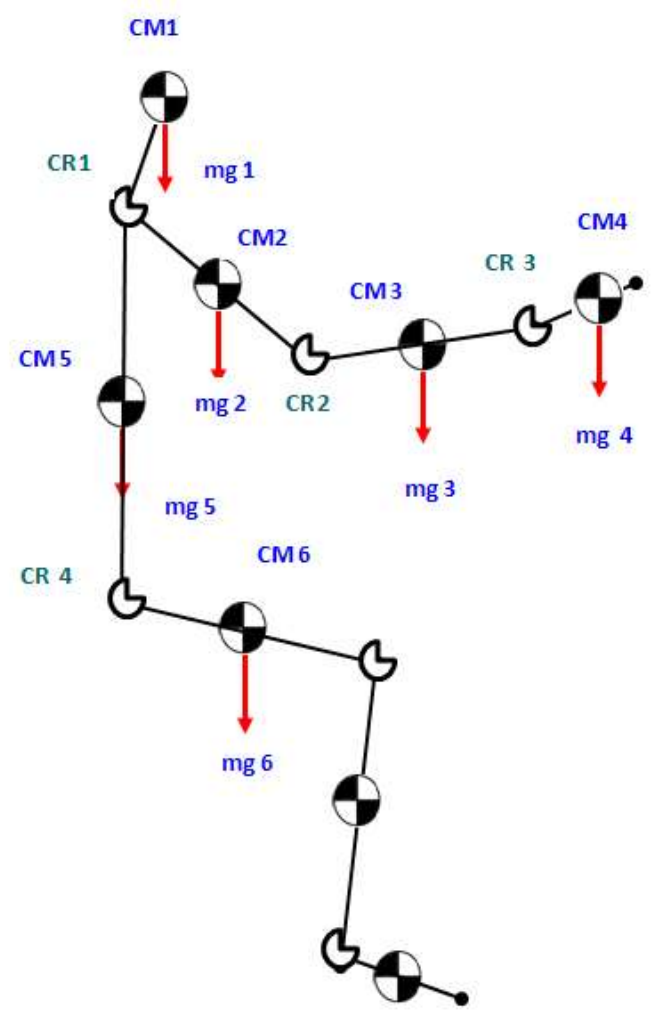

Figura No.2 Modelo Biomecánico Coplanar Estático para Escultura 
Las variables consideradas para ambos modelos son:

Longitud de segmento (Li): Esta variable es la distancia entre los centros de rotación que limitan a segmento, para fines de esta investigación, los segmentos considerados son el tronco, brazo, antebrazo, mano y cuello, para el caso de las posturas de pintura y grabado, agregándose otro segmento corporal más para el área de escultura, que corresponde al muslo. Las longitudes de los segmentos se derivan a partir de las marcas naturales del cuerpo con referencia a apoyos fijos. Se han empleado funciones matemáticas utilizando regresión lineal, teniendo como variables la estatura del cuerpo humano y la longitud de los huesos, de manera que pueden estimarse como percentiles de la población y como proporción de la estatura del cuerpo humano. En la figura No. 3 se representan estas valoraciones estadísticas las cuales fueron derivadas por Drillis y Contini (1966). Las ecuaciones de regresión que tienen como variable dependiente a la longitud de los huesos y como variable independiente la estatura fuero obtenidas por Webb Associates (1978) y en ellas el error estándar es 1 centímetro. Este error se incrementa ligeramente al obtener las longitudes de los segmentos a partir de la longitud de los huesos debido al error que se puede tener al calcular los centros de rotación entre segmentos adyacentes.

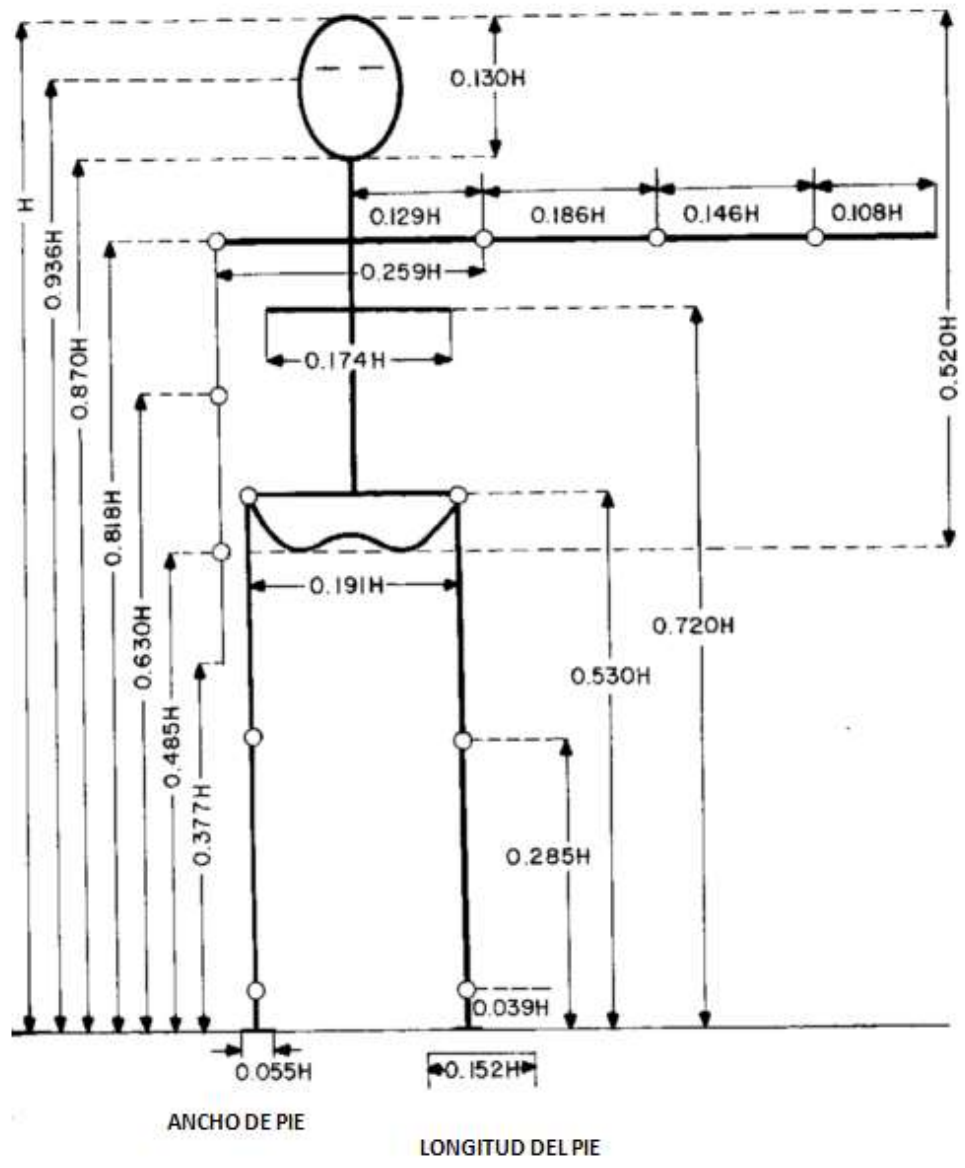


Figura No. 3 Valores estándares de longitudes de los segmentos (Recuperado de Ocuppational Biomechanics, John Willeys \&S Sons Inc, 1944, USA)

Centro de rotación (CRi): Es el punto de intersección de las proyecciones de los segmentos contiguos, denominados centros de rotación: cabeza-cuello, cuellotronco, codo, muñeca, cintura, rodilla, para los segmentos considerados en esta investigación.

Centro de masa (CMi): Es el lugar geométrico donde se concentra el efecto gravitacional de la distribución de la masa en el segmento. Una aproximación para establecer los centros de masa fue la proporcionada por Dempster (1955) en la que la localización del centro de mase se expresa como un porcentaje de la longitud de segmento, como se indica en la figura No. 4

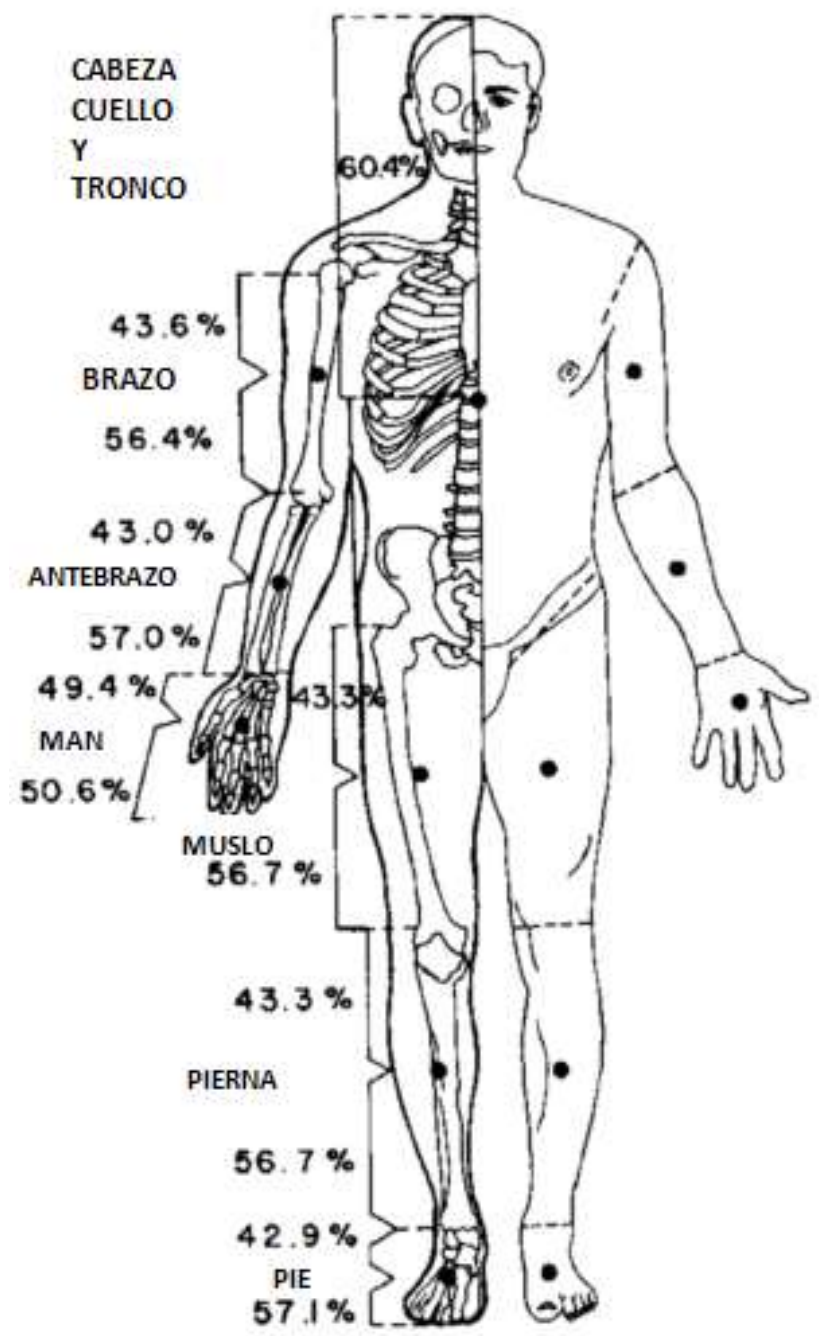

Figura No. 4 Estimación de los centros de masa de los segmentos corporales (Recuperado de Occupacional Biomechanics, John Willeys \& Sons, Inc., 1984, USA) 
Angulo de rotación de segmento $(\Theta i)$ : Es el ángulo de rotación de la línea central que une un centro de rotación con otro y que pertenece a un mismo segmento corporal, medido de la vertical del cuerpo en sentido de las manecillas del reloj.

Con base en el modelo biomecánico, para cada una de las tareas seleccionadas de pintura, escultura y grabado, se realizaron medidas de los ángulos superior e inferior de los segmentos corporales involucrados en ejecución de las tareas realizadas. Para determinar la normalidad de los datos de medición de ángulos de rotación de los segmentos corporales, se elaboraron los histogramas de cada una de las variables ángulo de rotación, se realizó prueba de bondad de ajuste KolmogorovSmirnov

Se empleó un goniómetro calibrado, una pantalla reticulada y una cámara fotográfica Nikkon de 20 Megapixeles para medir los ángulos de rotación en la postura de ejecución de la tarea y en la postura neutra., como se muestra en la figura No. 5

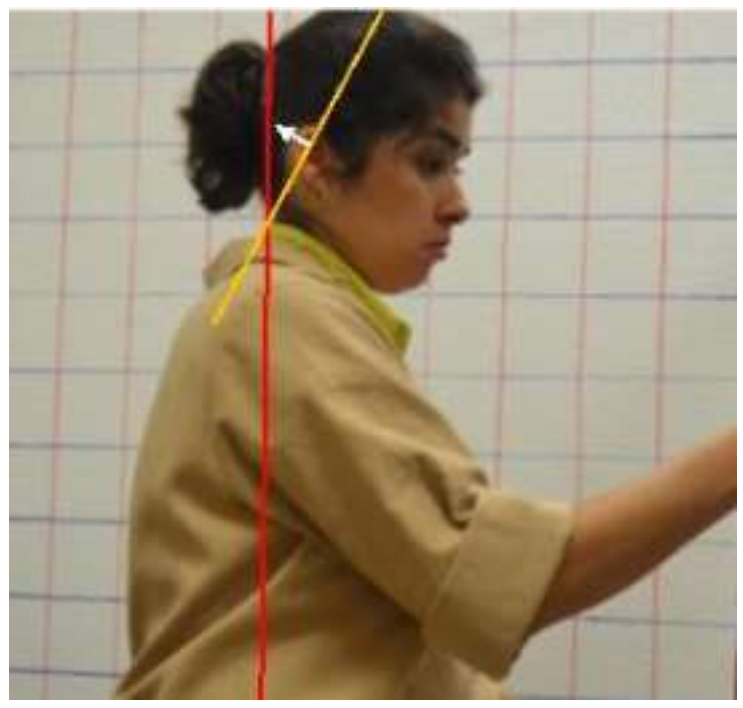

Figura No. 5 Esquema de medición de ángulos de rotación $\Theta i$

Peso de segmento corporal $\left(\mathrm{mg}_{\mathrm{i}}\right)$ : El peso del segmento corporal fue estimado a partir de la tabla No. 2 que indica las densidades en gramos por centímetro cúbico.

Tabla No.2 Densidades de segmentos corporales

\begin{tabular}{|l|l|l|}
\hline \multicolumn{1}{|c|}{ Segmento } & \multicolumn{1}{|c|}{$\begin{array}{c}\text { Densidad (Harless } \\
1860) \\
\mathrm{g} / \mathrm{cm} 3\end{array}$} & \multicolumn{1}{c|}{$\begin{array}{c}\text { Densidad } \\
(1955) \\
\mathrm{g} / \mathrm{cm} 3\end{array}$} \\
\hline Cabeza y cuello & 1.11 & 1.11 \\
\hline Tronco & - & 1.03 \\
\hline
\end{tabular}




\begin{tabular}{|l|l|l|}
\hline Brazo & 1.08 & 1.07 \\
\hline Antebrazo & 1.10 & 1.13 \\
\hline Mano & 1.11 & 1.16 \\
\hline Muslo & 1.07 & 1.05 \\
\hline Pierna & 1.10 & 1.09 \\
\hline Pie & 1.09 & 1.10 \\
\hline
\end{tabular}

Nota: Recuperado de Miller y Nelson, 1976 en Chaffin (1984)

\subsubsection{Repetibilidad y Reproducibilidad del Sistema de medición}

Se realizó un estudio de repetibilidad y reproducibilidad para el conjunto del sistema de medición a fin de comprobar la aleatoriedad de los errores en el sistema de medición. El estudio se realizó para los doce ángulos considerados, es decir, los seis para la posición real adoptada y los seis para la posición neutra.

2.2.4 Medición de Momentos de fuerza y cálculo valoración cuantitativa de riesgo de TME

A partir del modelo biomecánico definido para cada una de las tareas, se estimaron las longitudes de segmentos corporales a partir del modelo propuesto por Drillis y Contini (1966) y presentadas en kromer, Roebuck y Thompson (1975) se calcularon los momentos de fuerza para las posiciones de los segmentos corporales durante la ejecución de la tarea.

Se procedió a calcular la valoración cuantitativa e riesgo de TME el cual es un índice ponderado que contempla la sumatoria de momentos de fuerza ejercidos respecto al centro de rotación de la espalda baja en la postura de ejecución de la actividad y la sumatoria de momentos de fuerza ejercidos en postura neutra.

\subsubsection{Validación de valoración cuantitativa de riesgo de TME}

Se realizó validación del valor cuantitativo de riesgo de TME a través del proceso estadístico inferencial de prueba de hipótesis sobre la pendiente de la recta de regresión, con un nivel de significancia $\square=0.05$ para ambos valoraciones.

\subsection{Análisis de Datos y Procesamiento estadístico}


El procesamiento y análisis estadístico relativo a la información sociodemográfica de los participantes se describe en los siguientes apartados.

\subsubsection{Información Socio-demográfica de los participantes}

Fueron calculadas la media y la desviación estándar de la edad de los estudiantes participantes en el estudio, tanto para hombres como para mujeres. Se calcularon los porcentajes de estudiantes que tienen una relación laboral adicional a sus labores académicas.

\subsubsection{Evaluación RULA}

Se calculó el índice RULA para la totalidad de las evaluaciones realizadas y de acuerdo al procedimiento establecido por el método y en el programa señalado por la tabla No. 1.

\subsubsection{Valoración Cuantitativa de riesgo de TME}

Para cada una de las tareas seleccionadas, se realizó el cálculo de una valoración cuantitativa de riesgo de TME en función de las tareas desarrolladas. Esta valoración se estimó a partir de las variables descritas en el modelo biomecánico general.

2.3.4 Análisis estadístico de correlación índice RULA y valoración cuantitativa de riesgo de TME

Para cada uno de los 11 grupos de actividades de pintura, escultura y grabado, se calcularon los parámetros de la recta de regresión lineal, así como el índice de correlación correspondientes.

2.3.5 Análisis estadístico para la validación de la valoración cuantitativa de riesgo de TME

El análisis estadístico fue llevado a cabo usando la suite de software IBM SPSS (v24, SPSS Inc., Chicago, USA). Se realizó una prueba de hipótesis estadística para la recta de regresión lineal. La hipótesis nula para esta prueba es que la pendiente de la recta de regresión para el conjunto de valores índice RULA y valoración 
cuantitativa de Riesgo de TME es diferente de cero, el nivel de significancia usado para esta prueba es $\square=0.05$.

\section{Resultados}

\subsection{Participantes}

En este estudio participaron un total de 186 estudiantes de la licenciatura en Artes Plásticas de la Universidad de Sonora 136 mujeres y 50 hombres. El promedio de edad en mujeres fue de $19.13 \pm 1.23$ años y el promedio de edad en hombres 19.34 \pm 1.75 años. El porcentaje de mujeres con una relación laboral fue $13 \%$ y el porcentaje de hombre fue de $12 \%$. Todos los sujetos participaron en el proceso de evaluaciones y mediciones, durante la totalidad de los días y semanas en las cuales se llevó a cabo el estudio.

\subsection{Evaluación RULA}

La tabla No. 3 presenta el resumen de resultados obtenidos al realizar la evaluación RULA y obtener la valoración cuantitativa de riesgo de TME. Se registró un valor de índice RULA para todas las actividades realizadas de pintura, escultura y grabado en el $48.9 \%$ de las evaluaciones realizadas, este resultado implica que se recomienda estudiar y modificar de manera inmediata las posturas ejecutadas en las actividades evaluadas. Existe de acuerdo a el índice 7 un riesgo elevado de aparición de TME en las actividades evaluadas de las Artes plásticas. El nivel 6 de índice de riesgo se observa en todas las áreas, siendo el porcentaje de $18.4 \%$, para este resultado el método recomienda ampliar el estudio y modificar tan pronto sea posible las tareas. El $23.0 \%$ de las actividades de pintura, escultura y grabado presenta un índice con valor de 5 . Sólo el 9.7\% de las actividades presenta un índice RULA de 4, lo cual implica ampliar el estudio para una evaluación posterior. Ninguna de las actividades de las artes plásticas registró valores de índice RULA de 1,2 o tres que refieren a condiciones aceptables de las posturas realizadas por los ejecutantes de las artes plásticas.

Tabla 3 Resultados de Evaluación RULA

\begin{tabular}{ccccc}
\hline Indice Rula & \multicolumn{3}{c}{ Areas de Trabajo } & Porcentaje \\
\hline 7 & Pintura & Escultura & Grabrado & $48.9 \%$ \\
\hline 6 & Pintura & Escultura & Grabado & $18.4 \%$ \\
\hline 5 & Pintura & Escultura & Grabado & $23.0 \%$
\end{tabular}




\begin{tabular}{ccccc}
\hline 4 & Pintura & Escultura & Grabado & $9.7 \%$ \\
\hline 3 & - & - & - & $0.0 \%$ \\
\hline 2 & - & - & - & $0.0 \%$ \\
\hline 1 & - & - & - & $0.0 \%$
\end{tabular}

3.3 Valoración cuantitativa de Riesgo de TME

En tabla No. 4 se presentan los promedios de las valoraciones de riesgo de TME obtenidos para las tres áreas evaluadas:

Tabla No. 4 Valoraciones Cuantitativas de riesgo de TME por área e Indice RULA

\begin{tabular}{cccc}
\hline Índice RULA & \multicolumn{3}{c}{ Valoraciones } \\
& Pintura & Escultitura & Grabrado \\
\hline 7 & 1.88 & 1.78 & 1.86 \\
\hline 6 & 1.78 & 1.68 & 1.64 \\
\hline 5 & 1.47 & 1.51 & 1.54 \\
\hline 4 & & 1.49 & 1.6 \\
\hline
\end{tabular}

3.4 Análisis estadístico de correlación índice RULA y valoración cuantitativa de riesgo de TME.

En la tabla No. 5 se presenta el resumen de los índices de correlación encontrados entre las variables índice RULA e índice de riesgo de TME, así como el tipo de correlación encontrada para cada uno de los grupos evaluados en las áreas de pintura, escultura y grabado.

Tabla No. 5 Resumen de índices de correlación entre índice RULA y valoración cuantitativa de riesgo de TME

Grupo

Correlación de variables: Índice RULA y Valoración cuantitativa de Riesgo de TME 


\begin{tabular}{lcc}
\hline & Índice de Correlación $\mathrm{R}^{2}$ & Tipo de Correlación \\
\hline Pintura 1 & 0.258 & + \\
\hline Pintura 3 & 0.360 & + \\
\hline Pintura Específico 1 & 0.261 & + \\
\hline Pintura Específico 3 & 0.000 & + \\
\hline Escultura 1 & 0.000 & No determinada \\
\hline Escultura 3 & 0.385 & + \\
\hline Escultura Específico & 0.000 & No determinada \\
1 & & + \\
\hline Escultura Específico & 0.931 & + \\
3 & & + \\
\hline Grabado 1 & 0.152 & No determinada \\
\hline Grabado 3 & 0.092 & \\
\hline Grabado Específico & 0.000 & \\
1 & & \\
\hline
\end{tabular}

3.5 Validación de la valoración cuantitativa de Riesgo de TME

Se presenta en tabla No. 6 los resultados del análisis de varianza para probar la significancia estadística de la hipótesis Ho: Pendiente de la recta de regresión igual a cero, con un nivel de significancia de $\square=0.05$.

Tabla No. 6 Análisis de Varianza para la prueba de regresión lineal entre la valoración cuantitativa de riesgo de TME e índice RULA, nivel de significancia $\square=0.05$

\begin{tabular}{|c|c|c|c|c|}
\hline $\begin{array}{c}\text { Fuente de } \\
\text { Variación }\end{array}$ & $\begin{array}{c}\text { Suma de } \\
\text { Cuadrados }\end{array}$ & $\begin{array}{c}\text { Grados de } \\
\text { Libertad }\end{array}$ & $\begin{array}{c}\text { Media de } \\
\text { Cuadrados }\end{array}$ & Fo \\
\hline Regresión & 560.710 & 1 & 560.710 & 13871.0357 \\
\hline Error & 7.437 & 184 & 0 & \\
\hline
\end{tabular}


Dado que $\mathrm{Fo}=13871.0357$ es mayor que $\mathrm{F}=3.892$, se rechaza $\mathrm{Ho}$ con un nivel de significancia $\square=0.05$, por tanto, hay evidencia estadística para afirmar que existe una relación lineal entre la valoración cuantitativa de riesgo de TME y el índice de riesgo RULA. La implicación de este resultado es que el índice propuesto constituye una herramienta de prevención de riesgos de TME.

\section{Discusión}

Existen numerosos índices, los cuales se han desarrollado para evaluar el riesgo de trastornos músculo-esqueléticos, tales como RULA(McAtammey, 1993), REBA (Hignett, 2000) y LUBA (Doyhung, 2001) ninguno de estos métodos ha sido concebido para evaluar las condiciones de postura en las cuales se consideren como variables el peso del individuo y la estatura, así como las dimensiones de los segmentos corporales que están involucrados con las tareas que se ejecutan y de una manera particular con aquellas de las artes plásticas. EL método RULA evalúa tareas de tipo continuo y repetitivo, las cuales también son comunes durante la ejecución de las actividades de las artes plásticas. El método LUBA es un procedimiento que permite obtener un índice de no confort para los movimientos de la mano, brazo, cuello y espalda en posiciones estáticas, el nivel de no confort se obtiene por la suma de las desviaciones angulares respecto de la neutral, en nuestra investigación, el número índice propuesto es un índice agregado, se obtiene de manera cuantitativa sumando los momentos de fuerza que se generan en respecto al centro de rotación de la espalda baja y dividiéndolo por los momentos de fuerza que se generan en la posición neutra, tomando en cuenta variables como son el peso y la talla del individuo. REBA es un método de análisis de la postura adecuado a tareas que implica cambios inesperados de postura mientras se realiza manipulación de cargas inestables. Su aplicación nos permite conocer el riesgo de lesiones asociadas a las posturas. Las metodologías RULA, REBA y LUBA son herramientas de gran valor para realizar las evaluaciones por ser rápidas y de fácil ejecución. En esta investigación la valoración cuantitativa de riesgo de TME suministra una evaluación numérica cuantitativa basada en el modelo biomecánico coplanar empleado para el cálculo de momentos de fuerza respecto al centro de rotación de la espalda baja.

La valoración cuantitativa de riesgo de TME es consistente con el índice RULA obtenido en el estudio de campo preliminar conducido en las 186 tareas evaluadas, es decir, para la puntuación 7 de índice RULA, la valoración de riesgo de TME presentó un promedio de 1.84, que implica que la tarea debe modificarse y que hay un alto riesgo de desarrollo de TME. Para la puntuación 6 de RULA, la valoración de riesgo de TME fue en promedio de 1.7, la recomendación para este caso es que 
existe riesgo de desarrollo de TME y se debe ampliar el estudio. Para la puntuación de 5 en el índice RULA, el promedio de valores asociados de valoración cuantitativa de riesgo de TME, para el cual sólo se recomienda ampliar el estudio. Finalmente para el valor 4 de índice RULA, la valoración de riesgo de riesgo de TME registra un valor de 1.54 en promedio, en este caso sólo se recomienda ampliar el estudio. Los valores del índice RULA y valoración cuantitativa de riesgo de TME fueron sometidos a un análisis de regresión lineal, y los resultados revelaron que en el $90 \%$ de los casos existe una correlación positiva, sin embargo, el índice de correlación es bajo en todos los casos, los cuales aluden a que no es posible que la tarea se ejecute siempre con los mismos movimientos de manera exacta.

\section{Conclusiones}

Con gran cantidad de investigación enfocada en los trastornos músculo esqueléticos en los diferentes sectores profesionales, que establecen una relación muy grande entre los riesgos de aparición de trastornos músculo esqueléticos y las posturas corporales, repetición de movimientos y cargas sostenidas. Nuestra investigación muestra que es posible determinar el riesgo de aparición de TME, considerando las características individuales de los sujetos, como son el peso y la estatura. Las valoraciones de riesgo de TME obtenidos a través del método cuantitativo que se propuso son consistentes con el valor obtenido de índice RULA. Las estimaciones individuales de los índices de correlación revelaron una correlación positiva entre las dos variables en el $90 \%$ de los casos, sin embargo, el índice de correlación entre los mismos es bajo. Los valores de índice de correlación bajos pueden estar relacionados al hecho de que la actividad bajo análisis no se lleva a cabo exactamente con los mismos movimientos del cuerpo, varía entre los individuos como en el individuo mismo al repetirse. El aporte de esta investigación radica en que la valoración cuantitativa propuesta considera las variables antropométricas individuales los individuos a ser evaluados, considerando las posturas angulares de los segmentos del cuerpo y los pesos asociados a cada segmento, los cuales son particulares para cada individuo, por tanto, podríamos considerarlo más robusto que los métodos de evaluación cualitativos conocidos. Sin embargo, se considera que el método propuesto requiere de muchos recursos, además de cálculos que en conjunto consumen tiempo y energía del evaluador. Existen otros elementos a considerar, como son la repetibilidad y reproducibilidad de los instrumentos usados para medir valores de ángulos de rotación de los segmentos, la cual debe ser verificada cada vez que se inicien procesos de medición. A futuro, se visualiza una oportunidad para realizar un proceso de reconocimiento de patrones, utilizando los datos obtenidos como conjunto de entrenamiento y así realizar diagnósticos previos a la aparición de un trastorno músculo esquelético, en favor de su predicción no de su detección. 


\section{Referencias}

Amis, A., Dowson, D., Wright, V. (1980). Analysis of Elbow Forces Due to highSpeed Forearm Movements. Biomechanics 13. 825-831.

An, K.N., Chao, E.Y., Cooney, W.P., Linacheid, R.L. Normative Model of Human hand for biomechanical Analysis. Journal of Biomechanics 7, 343-348.

Ayoub, M.M., Mital, A., Bakken, G.M., Asfour, S.S., Bethea, N. J. (1974).

Development of Strenght and Capacity Norms for Manual Materials Handling. Human Factors 22(3).35-51.

Ayoub, M.M, El-Bassousi, M.M. (1976). Dynamic Biomechanical Model for Saggital Lifting activities . Proceedings of the $6^{\text {th }}$ Congress of International Ergonomis Association. Human Factors. 355-359.

Breniere, Yvon, Ribreau, Christian. (1998). A double-inverted pendulum model for studying the adaptability of postural control to frequency during human stepping in place. Biological Cybernetics, 79. 337-345.

Bruce P. Bernard. (1997). Musculoskeletal Disorders and Workplace Factors: A Critical Review of Epidemiologic Evidence for Work Related Musculoskeletal Disorders of the Neck, Upper Extremity, and Low Back; Department of Health and Human Services, National Institute for Occupational Safety and Health, Cincinnati, $\mathrm{OH}$.

Burstein, A.H., Shaffer, B. W., Frankel, V.H., Elastic Analysis of Condylar Structures. American Society of Mechanical Engineers Paper 70-WA/BHF-1.

Bresler, B., Frankel, J.P. (1950). The Forcesand momentsin the leg during level walking. Transactions of the American Society of Mechanical Engineers. 27-30.

Caban-Martinez , J. , Lee, David J. , Clarke C. Tainya, Davila, Evelyn P., Clark III, John D, Ocasio A. Manuel, Fleming Lora E. (2010). Self-Reported Joint and Back pain among Hispanic construction workers: A pilot workplace musculoskeletal assessment. Journal of Musculoskeletal Research, Vol. 13, No. 2, 49-55.

Caillet, R. (1968). Low Back Pain Syndrome. EE.UU.:Davis Co.

Cappozo, A., Leo, T., Pedotti, A. (1975). A General Computing Method for the Analysis of Human Locomotion. Journal of Biomechanics 8:307-320.

Chaffin, Don B, Andersson, Gunnar B.J. (1984). Occupational Biomechanics. EE. UU.: John Willeys \& Sons, Inc.

Chaffin, Don B., Baker, W.H., (1970). A Biomechanical Model for Analysis of symmetric saggital plane Lifting. American Institute of Industrial Engineering Transactions 2. 16-27.

Corlett, E. N. and Bishop, R. P. (1976). A Technique for Assessing Postural Discomfort. Ergonomics 19(2). 175-182.

Crowninshield, R. D. (1978). Use of optimization Techniques to Predict Muscle Forces. Journal of Biomechanic Engineering 100. 88-92.

De Looze P. Michiel, Kuijt-Evers, Lottie, Van Dieen Jaap. (2003). Sitting comfort and discomfort and the relationships with objective measures; Ergonomics Augus, 2003, Vol. 46, No. 10. $985-997$. 
Dempster, W. T.(1955). Space requirements of the seated operator. WADC-TR-5159, Aerospace Medical Research Laboratories. 67-78.

De Luca, C.J., Forrest, W.J., Force Analysis of Individual muscles acting simultaneously on the shoulder joint during isometric abduction. Journal of Biomechanics. 385-393.

D.M. Rempel, L. Punnett. (1977). Epidemiology of wrist and hand disorders, in: M. Nordin, G.B. Andersson, M.H. Pope (Eds.), Mosby-Year Book, Inc, Philadelphia, PA, 421-430.

Dohyung, Kee;Waldemar, Karwowski. (2001). LUBA; an assessment technique for postural loading on the upper base don joint motion discomfort and máximum holding time; Applied Ergonomis 32. 357-366.

Drillis, R., R. Contini, (1966).Body segment parameter. New York University, New York: BP 174-945, Tech. Rep. No.1166.03, School of engineering and science.

Engin, A. E., Korde, M.S. (1974). Biomechanics of Normal and Abnormal Knee Joint. Journal of Biomechanics 7:325-334.

Frievalds,A. D., Chaffin, B., Garg, A., Lee, K. (1984). A Dynamic Biomechanical Evaluation of Lifting Maximum Acceptable Loads. Journal of Biomechanics 17(4). 251-262.

Frievalds, A. (1984); Computarized-aided Strenght Prediction Using the Articulated Total Body Model. Computer and Industrial Engineering 8 (2). 107-118.

Froines, John R. Phd, Dellenbauch, Cornelia A. Mph and Wegman, David H. Md. (1986). Occupational Health Surveillance: A Means to Identify Work-Related Risks. American Journal of Public Health Vol 76 No. 9.

Gruver, W.A., Ayoub, M.A., Muth, M.B. (1979). A Model for Optimal Evaluation of Manual Lifting Tasks. Journal of Safety Research 11. 61-71.

Hatze, H. (1976). The Complete Optimization of a Human motion. Mathematical Bio-sciences 28. 99-135.

Hignett, S.; McAtamney, L. (2000). Rapid Entire Body Assessment: Reba. Applied Ergonomics, 3, 201-205.

Instituto Mexicano del Seguro Social (2009). Estadísticas Institucionales del IMSS. Coordinación de Salud en el trabajo. México. Recuperado 28 agosto 2014, http://www.e-

mexico.gob.mx/wb2/emex/emex_estadisticas_institucionales_del_IMSS Juno Natarén, Janthé, Noriega Elío Mariano; Los trastornos musculoesqueléticos y la fatiga como indicadores de las deficiencias ergonómicas y en la organización del trabajo; Salud de los Trabajadores, ISSN-e 1315-0138, Vol. 12, №. 2, 2004 , págs. 27-41

Kattan, A., Nadler, G. (1970). Equations of Hand Motion Path for Work space Design. Human Factors 11. 123-130.

KettlelKamp, D.B., Chao, E.Y. (1972). A Method for Quantitative Analysis of medial and lateral compression Forces at the Knee during Standing. Clinical Orthopedic Relative Research83. 202-213.

Kölsch, M., Beall, A. and Turk, M. (2003). An Objective Measure for Postural Comfort. In Proc. HFES 47 th Annual Meeting this publication, 45-67. 
Kroemer, Karl H.E., Snook, Stover H., Meadows, Susan K. (1988). Ergonomic Models of Anthropometry, Human Biomechanics, and Operator-equipment interfaces, Proceedings of a workshop; Stanley Deutsch Editors. 19-42.

Kroemer, H.E., Roebuck, J.A., Thomson, W.G. (1975). Engineering Anthropometry Methods, Wiley-Interscience, New York, 77-128.

Lanzotti, A. Trotta M., Vanacore, A. (2011). Validation of a new index for seat comfort assessment based on objective and subjective measurements. Proceedings of the IMProVe 2011 International conference on Innovative Methods in Product Design June 15th-17th, 35-45.

Lin, H.S., Liu, Y.K., Ray, G., Nikravesh, P. (1978). System Identification for Material Properties of the Intervertebral Joint. Journal of Biomechanics 11, 1-14.

LL. Z. Zhang, M. G. Helander, Drury C. G. (1996). Identifying factors of comfort and discomfort in sitting. Human Factors 38, 377-389.

MacConaill, M.A. (1967). The Ergonomic Aspects of Muscular Mechanics. Pp. 6980 in Evans, F.G. Study on the Anatomyand Function of Bones and Joints. Berlin:Springer-Verlag.

Mahboobin,A.; Beck, C.L., . Moeinzadeh, M.H, Loughlin, P. (2002). Analysis and Validation of A Human Postural Control Model. Proceedings of the American Control Conference Anchorage, 56-67.

Marley, Robert J., Kumar, Nirmal. (1996). An improved musculoskeletal discomfort assessment tool; International Journal of Industrial Ergonomics 17, 21-27.

McAtamney, L., Corlett, E.N. (1993). Applied RULA: a survey method for the investigation of work-related upper limb disorders. Applied Ergonomics, 24, 91-99. Micheau, Philippe, Kron, Aymeric, Bourassa, Paul, Bourassa; Evaluation of the lambda model for human postural control during ankle strategy; Biologycal Cybernetics, 89, 227-236.

Miller, D. I., R.C. Nelson. (1976). Biomechanics of Sport, Philadelphia, EE. UU.: Lea and Febiger.

Minna, R.J. (1981). Forces at the Knee Joint: Anatomical Considerations. Journal of Biomechanics 14, 633-643.

Mitropoulos Panagiotis, Namboodiri Manoj, (2011).New Method for Measuring the Safety Risk of Construction Activities: Task Demand Assessment; Journal of construction Engineering and Management, 30-38.

Moes, Niels CCM. (2005). Analysis of sitting discomfort a Review, Contemporary Ergonomics, 202-204.

Morrison, J.B. (1969). Function of the Knee joint in various activities. Bio-Medicine Engineering 4, 573-580.

Pearson, J. R., McGinley,D.R. (1961). Dynamic Analysis of the Upper Extremity for Planar Motions. University of Michigan Tech Report 04468, 55-89.

Punnet, Laura, Wegman, H. David. (2004). Work-related musculoskeletal disorders: the epidemiologic evidence and the debate. Journal of Electromyography and Kinesiology 14, 13-23.

Tahbouba, Karim, Mergnerb, Thomas. (2007). Biological and engineering approaches to 
human postural control. Integrated Computer-Aided Engineering 14, 15-31. Rajaram K. Manoharan, Mary L. Bouxsein. (2010). A biomechanical model for estimating loads on thoracic and lumbar vertebrae. Clinical Biomechanics 25, 853858.

Rodgers, M. Mary, Cvanagh, R. Peter. (1984). Glossary of Biomechanical Terms, Concepts, and Units. Physical Theraphy, 64-87.

Seireg, A., Arvikar, R. (1975). The prediction of Muscular Load Bearing and Joint Forces in the Extremities. Journal of Biomechanics 8, 89-102.

Schultz, A.B., Anderson, G.B.J. (1981). Analysis of Loads on the lumbar spine. Spine 6(1), 76-82.

Schultz, A.B., Anderson, G.B.J, Haderpecck, K., Ortengren, R.,Nordin, M., Bjork, R. (1982). Analysis and Measurement of Lumbar Trunk load in Tasks Involving Bends and Twists. Journal of Biomechanics 15, 669-675.

Slote, L., Stone, G. (1963). Biomechanical Power Generated by Forearm Flexion. Human Factors 5,433-452.

Sonne Michael, Villalta, Dino L., Andrews, David M. (2012). Development and evaluation of an office ergonomic risk checklist: ROSA: Rapid office strain assessment; Applied Ergonomics 43, 98-108.

Villiapan S, Sevensson NL, Wood RD. (1977). Three dimensional tress analysis of the human femur, Com Biol Med 7, 253-264.

Webb Associates. Anthropometric Source Book. (1978). EE. UU.: NASA Ref.1024, Nat'l Aero-Space Administration.

Wismans, J., Veldpau, F., Janssen, J. Huson, A.,Struben, P. (1980). A threedimensional mathematical model of the knee-joint. Journal of Biomechanics 13; 677686.

Yeo, B.P. (1976). Investigations Concerning the Principle of Minimal Total Muscular Force. Journal of Biomechanics 9, 413-416.

Cómo citar este artículo:

Sortillón González, P. E. ., Ortega Encinas, L. U. ., López Bojórquez, J. S., LeyvaPacheco, J. A. ., \& Ochoa Salcido, G. (2021). Validación de un método cuantitativo de Evaluación de Riesgos de Trastornos Músculo-esqueléticos en las tareas de las artes plásticas a partir de un método cualitativo. Revista De Investigación Académica Sin Frontera: División De Ciencias Económicas Y Sociales, (35). https://doi.org/10.46589/rdiasf.vi35.338 https://revistainvestigacionacademicasinfrontera.unison.mx/index.php/RDIASF/article/view/338 


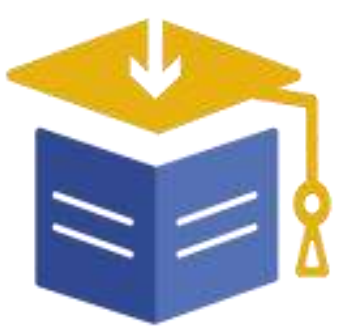

อ

\section{CRIS - UNISON Sistema de Gestión de la Investigación}

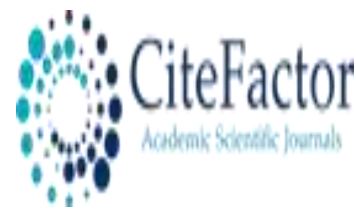

Neliti - Indonesia's Research Repository

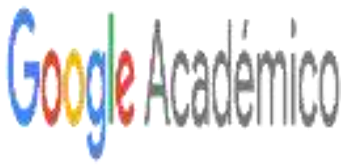

๑ Dialnet

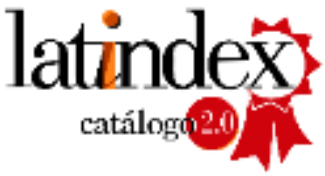

DORA

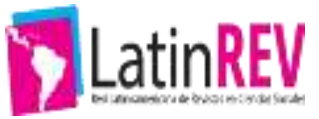

REDIB

Fied Iberoamanican
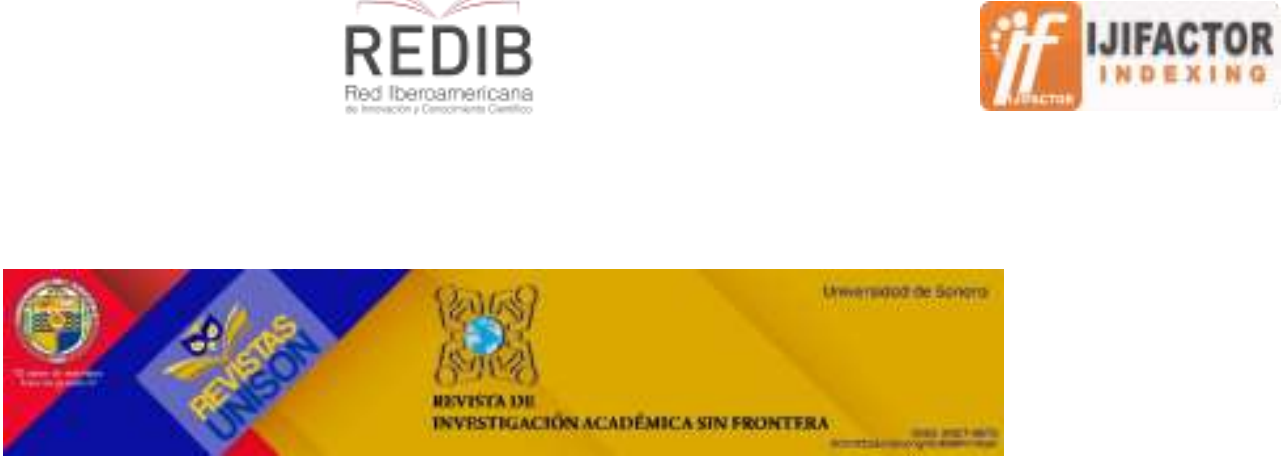